\title{
ANÁLISE DA DENSIDADE MINERAL ÓSSEA EM PACIENTES COM FENILCETONÚRIA E SUA CORRELAÇÃO COM PARÂMETROS NUTRICIONAIS
}

\author{
BONE MINERAL DENSITY ASSESSMENT IN PATIENTS \\ WITH PHENYLKETONURIA AND ITS CORRELATION WITH \\ NUTRITIONAL PARAMETERS
}

Clin Biomed Res. 2019;39(1):24-31

1 Programa de Pós-graduação em Ciências Médicas, Endocrinologia, Universidade Federal do Rio Grande do Sul (UFRGS). Porto Alegre, RS, Brasil.

2 Serviço de Genética Médica, Hospital de Clínicas de Porto Alegre (HCPA). Porto Alegre, RS, Brasil.

3 Serviço de Nutrição e Dietética, Hospital de Clínicas de Porto Alegre (HCPA). Porto Alegre, RS, Brasil.

4 Center for Individualized Medicine, Mayo Clinic. Rochester, Minnesota, Estados Unidos.

5 Department of Clinical Genomics, Mayo Clinic. Rochester, Minnesota, Estados Unidos.

6 Programa de Pós-graduação em Genética e Biologia Molecular, Universidade Federal do Rio Grande do Sul (UFRGS). Porto Alegre, RS, Brasil.

7 Departamento de Genética, Universidade Federal do Rio Grande do Sul (UFRGS). Porto Alegre, RS, Brasil.

Autor correspondente: Ida Vanessa Doederlein Schwartz idadschwartz@gmail.com Hospital de Clínicas de Porto Alegre (HCPA)

Rua Ramiro Barcelos, 2350

90035-903, Porto Alegre, RS, Brasil.

\section{RESUMO}

Introdução: Redução da densidade mineral óssea (DMO) está associada à Fenilcetonúria (PKU), mas a causa desta associação não é completamente entendida. O objetivo desse estudo foi avaliar a ingestão de nutrientes relacionados ao metabolismo ósseo (cálcio, fósforo, magnésio, potássio), e sua associação com a DMO em pacientes com PKU.

Métodos: Estudo transversal, observacional. Foram incluídos 15 pacientes (PKU Clássica=8; Leve=7; mediana de idade $=16$ anos, $I Q=15-20$ ), todos em tratamento com dieta restrita em fenilalanina (Phe) e 13 em uso de fórmula metabólica. Foi realizado recordatório alimentar de 24 horas de um dia e demais dados (histórico de fraturas, parâmetros antropométricos, DMO e níveis plasmáticos de Phe, Tyr, cálcio) foram obtidos por revisão de prontuário.

Resultados: Nenhum paciente apresentou histórico de fraturas e seis realizavam suplementação de cálcio (alteração prévia da $\mathrm{DMO}=5$; baixa ingestão=1). A mediana dos níveis de Phe foi $11,6 \mathrm{mg} / \mathrm{dL}(\mathrm{IQ}=9,3-13,3)$. Em relação ao recordatório alimentar, dez indivíduos apresentaram inadequado consumo de carboidratos; 14, de lipídeos; 9, de cálcio; 11, de magnésio; 13, de fósforo; e todos de potássio. A mediana da DMO foi de $0,989 \mathrm{~g} / \mathrm{cm}^{2}(\mathrm{IQ}=0,903-1,069)$, sendo duas classificadas como reduzidas para idade, ambas de pacientes com PKU Leve que recebiam suplementação de cálcio. Não foi observada correlação entre níveis de Phe, DMO e demais variáveis analisadas.

Conclusão: Redução da DMO não foi frequente na amostra, embora ingestão inadequada de cálcio assim o seja. Estudos adicionais são necessários para esclarecer o efeito da Phe e da ingestão dietética sobre o metabolismo ósseo na PKU.

Palavras-chave: Fenilcetonúria; fenilalanina; densitometria; densidade óssea; terapia nutricional

\section{ABSTRACT}

Introduction: Reduced bone mineral density (BMD) is associated with phenylketonuria (PKU), but this association is not completely understood. This research aimed to evaluate intake of nutrients related to bone metabolism (calcium, phosphorus, magnesium, potassium) and its association with BMD in patients with PKU.

Methods: In this cross-sectional, observational study, 15 patients with PKU (Classical=8, Mild=7; median age=16 years, $I Q=15-20$ years) were included, all of them on phenylalanine (Phe) restricted diet and 13 being supplemented with a metabolic formula. A 24-hour dietary recall was performed and remaining data (history of fractures, 
anthropometric parameters, BMD and plasma Phe, tyrosine and calcium levels) were obtained through medical chart review.

Results: No patient had any fractures and six received calcium supplements, five due to previous change in BMD and one due to inadequate nutritional intake. Median Phe level was $11.6 \mathrm{mg} / \mathrm{dL}(\mathrm{IQ}=9.3-13.3)$. In relation to dietary recall, all individuals had inadequate intake of some nutrient (carbohydrate=10; lipids $=14$; calcium=9; magnesium=11; phosphorus=13; potassium=15). The median BMD was $0.989 \mathrm{~g} / \mathrm{cm}^{2}$ $(I Q=0.903-1.069)$. Two cases were classified as low BMD for age, both in patients with mild PKU receiving calcium supplements.

Conclusion: Reduced BMD was not common in this sample, although inadequate calcium intake was frequently reported. Additional studies are needed to clarify the effect of Phe and dietary intake on bone metabolism in patients with PKU.

Keywords: Phenylketonuria; phenylalanine; densitometry; bone density; nutrition therapy

A Fenilcetonúria (PKU) é um erro inato do metabolismo de herança autossômica recessiva no qual ocorre redução da atividade da enzima fenilalanina hidroxilase, responsável pela conversão do aminoácido fenilalanina (Phe) em tirosina (Tyr), levando ao aumento dos níveis plasmáticos de $\mathrm{Phe}^{1}$. O tratamento de primeira escolha é dietético, sendo necessária a restrição de fontes proteicas de origem animal e de algumas fontes proteicas de origem vegetal que contenham maior teor de Phe, além do uso de fórmula metabólica (FM), fonte de vitaminas, minerais e aminoácidos, com exceção de Phe. O quadro clínico de pacientes não tratados é caracterizado por alterações no desenvolvimento neuro-psicomotor, déficit cognitivo, padrão autista de comportamento, transtorno do espectro autista, convulsões, irritabilidade e alterações na densidade mineral óssea $(\mathrm{DMO})^{2}$. O tratamento deve ser iniciado imediatamente após o diagnóstico e deve ser realizado durante toda a vida, a fim de prevenir sequelas ou o desenvolvimento do quadro clínico ${ }^{3,4}$.

Deficiências nutricionais foram observadas em indivíduos com PKU devido à dieta restrita, sendo o substituto proteico responsável por suprir parcial ou até totalmente as necessidades dos indivíduos, sem que quantidades excessivas de Phe sejam ingeridas ${ }^{5-8}$. Por outro lado, Evans et al..$^{9}$ demonstraram que indivíduos com PKU em uso de FM e com ingestão de micronutrientes acima do valor recomendado, apresentaram concentração plasmática reduzida desses micronutrientes, decorrente da baixa biodisponibilidade que a FM apresenta.

Alterações na DMO têm sido observadas em pacientes com $\mathrm{PKU}^{2}$. Crianças com PKU acima de 8 anos com níveis séricos de Phe $<1200 \mathrm{mmol} / \mathrm{L}$ apresentaram menor Score Z $(-0,04 \pm 0,44)$ quando comparadas a crianças com níveis $>1200 \mathrm{mmol} / \mathrm{L}(-0,56 \pm$ $0,78)^{10}$. Em outro estudo realizado com 31 pacientes com PKU, com média de idade de $25 \pm 5,3$ anos, foi observada a presença de osteoporose $(6,5 \%) \mathrm{e}$ osteopenia ( $38,7 \%)$, sendo que $32,2 \%$ dos pacientes apresentaram níveis de Phe séricos dentro do valor alvo em tratamento, não demonstrando correlação entre níveis séricos de Phe e DMO ${ }^{11}$.

Estudos ainda mostraram que pacientes com PKU aderentes ao tratamento apresentaram redução dos níveis plasmáticos de vitamina $\mathrm{D}$, cálcio, magnésio e selênio, por outro lado, foram observados níveis plasmáticos de fósforo, zinco, cobre e folato normais ${ }^{12,13}$. Concentrações plasmáticas de fósforo mais elevadas foram observadas em indivíduos com PKU e alteração da DMO quando comparados a indivíduos com PKU e adequada $\mathrm{DMO}^{14}$.

Neste sentido, este estudo tem por objetivo avaliar a DMO e sua correlação com parâmetros nutricionais e níveis de Phe sérica em pacientes com PKU acompanhados em um centro de referência do Rio Grande do Sul.

\section{MÉTODOS}

Este é um estudo transversal, observacional, com amostragem por conveniência, aprovado pelo Comitê de Ética do Hospital de Clínicas de Porto Alegre (CEP/HCPA). O recrutamento dos pacientes ocorreu de março a outubro de 2016. Todos os participantes ou os seus responsáveis assinaram o termo de consentimento livre e esclarecido. Os pacientes incluídos possuíam diagnóstico bioquímico confirmado de PKU; estavam em tratamento no Ambulatório de Distúrbios Metabólicos do Serviço de Genética Médica do Hospital de Clínicas de Porto Alegre (ADM-SGM/HCPA), Brasil; e realizaram absorciometria de raios- $x$ de dupla energia (DEXA) em um intervalo máximo de 1 ano antes do consentimento de participação no estudo. 
A presença de comorbidades associadas e/ou uso de medicações não relacionadas à dietoterapia específica com possibilidade de interferir no metabolismo ósseo foram considerados critérios de exclusão.

$\mathrm{O}$ acompanhamento dos pacientes com PKU do ADM-SGM/HCPA é realizado com frequência de 3 a 6 meses. O controle metabólico é avaliado em cada consulta por meio dos valores plasmáticos de Phe e Tyr. Os valores alvo de Phe em tratamento por faixa etária são classificados de acordo com o Protocolo Clínico e Diretrizes Terapêuticas para $P K U^{15}$, sendo considerados valores permitidos de 2-6 mg/dL (120-360 mmol/L) para pacientes de 1 a 13 anos e entre $2-15 \mathrm{mg} / \mathrm{dL}(120-900 \mathrm{mmol} / \mathrm{L})$ para pacientes maiores de 13 anos. O sistema ósseo é avaliado anualmente através da dosagem sérica de cálcio, fosfatase alcalina óssea e vitamina D. Enquanto radiografia simples e/ou DEXA são realizadas a cada 2 anos ou menos, a depender das circunstâncias clínicas.

Os pacientes foram classificados quanto ao tipo de PKU de acordo com os critérios estabelecidos por Nalin et al. ${ }^{16}$. Os dados antropométricos e dietéticos foram aferidos e coletados no momento da inclusão. Aingestão alimentar de macronutrientes e micronutrientes foi avaliada por meio de um recordatório alimentar de 24 horas, enquanto o cálculo do consumo diário total dos nutrientes, sem considerar a ingestão de suplementos, foi realizado por meio do software Nutwin ${ }^{\circledR}$ versão 1.5. A adequação da ingestão alimentar foi classificada de acordo com as Recommended Dietary Allowances (RDA) estabelecidas pela Dietary References Intakes (DRIs) em 201117. Quando não disponíveis valores para RDA, foram utilizados os pontos de corte de Adequate Intake (AI). O peso foi aferido com os pacientes vestidos com roupas leves, de braços eretos, pés dispostos no centro da balança e braços estendidos em balança digital (Filizola ${ }^{\circledR}$ ) com precisão de $0,1 \mathrm{~kg}$. A estatura foi aferida com o paciente descalço, pés paralelos, tronco ereto, braços estendidos e a cabeça de maneira que a órbita ocular esteja no mesmo plano que o orifício externo da orelha, conforme Plano de Frankfort em estadiômetro de parede com precisão de $0,1 \mathrm{~cm}$. Estas medidas foram utilizadas para o cálculo do índice de massa corporal (IMC) $\mathrm{kg} / \mathrm{m}^{2}$.

Os dados referentes às DEXAs e parâmetros bioquímicos foram obtidos por meio de revisão de prontuário. Para a avaliação dos parâmetros bioquímicos séricos de Phe e Tyr, realizados por método de cromatografia líquida de alta eficiência (HPLC), e dosagem de cálcio sérico realizada por método colorimétrico orto-cresolftaleína foram considerados os exames coletados até um ano antes da realização da DEXA. Para aqueles pacientes com mais de uma dosagem plasmática de Phe, Tyr e cálcio realizadas dentro do período estabelecido, foi utilizada a mediana destes valores para posterior análise. Quanto aos dados relativos à DMO por DEXA, foram considerados os resultados mais recentes, estabelecendo um intervalo máximo de um ano antes da data de inclusão no estudo. Os achados de DEXA foram classificados de acordo com critérios da Sociedade Brasileira de Densitometria Clínica ${ }^{18} \mathrm{em}$ escore $Z$ ou $T$, tendo em vista o sítio ósseo avaliado por faixa etária.

Para análise da normalidade das variáveis foi utilizado o teste de Shapiro-Wilk, pelo qual as mesmas foram classificadas como não-paramétricas. Os dados foram expressos em medianas e intervalos interquartis e o teste de Wilcoxon-Mann-Whitney foi utilizado para comparação entre as medianas. As correlações foram analisadas pelo coeficiente de correlação de Spearman. A análise estatística foi realizada utilizando o programa Statistical Package for Social Sciences versão $18^{\circledR}$ (SPSS Inc., Chicago, IL), com valor de significância de $p$ igual ou inferior a $5 \%$.

\section{RESULTADOS}

Durante o período de realização do estudo 82 pacientes com diagnóstico de PKU estavam sendo atendidos pelo ADM-SGM/HCPA; 65/82 consultaram no período e, destes, apenas 15 (PKU Clássica $=8$, PKU Leve $=7$, sexo feminino $=8$, mediana de idade $=16, I Q$ 25-75=15-20 anos, amplitude $=8-28$ anos) preenchiam os critérios de inclusão (32 não tinham realizado DEXA, 15 haviam realizado DEXA há mais de um ano e 3 apresentaram algum critério de exclusão). Apenas um indivíduo era pré-puberal. Todos os quinze pacientes incluídos estavam em dieta restrita em Phe, treze realizavam suplementação de aminoácidos essenciais e micronutrientes (ou seja, usavam fórmula metabólica) e seis realizavam suplementação oral de cálcio, cinco devido à alteração prévia da DMO e um devido à baixa ingestão do nutriente.

A mediana dos níveis séricos de Phe do ano anterior ao da realização da DEXA foi de $11,6 \mathrm{mg} / \mathrm{dL}$ (IQ 25-75=9,3-13,3), estando um paciente fora do valor alvo de Phe para sua faixa etária, sendo a mediana de idade de 16 anos (IQ 25-75=15-20). Os níveis séricos de Tyr e de cálcio apresentaram mediana de $0,8 \mathrm{mg} / \mathrm{dL}(\mathrm{IQ} 25-75=0,6-1,2)$ e de 9,7 mg/dL (IQ 25-75=9,5-9,9), respectivamente.

A mediana do intervalo entre a realização das DEXAs e dos recordatórios alimentares foi de 4 meses (IQ 25-75=2-9). A mediana das DMOs foi de $0,989 \mathrm{~g} / \mathrm{cm}^{2}$ (IQ 25-75=0,903-1,069), sendo que dois pacientes apresentaram baixa DMO para idade (ambos com PKU Leve, idade de 14 e 15 anos, 
Phe de 10,9 mg/dL e 5,2 mg/dL, respectivamente). Os grupos PKU Clássica e PKU Leve foram comparados quanto às medianas das DMOs e aos parâmetros bioquímicos de Phe, Tyr e cálcio séricos, não sendo observada diferença entre os grupos conforme descrito na Tabela 1.

Em relação aos parâmetros antropométricos foram analisados peso, com mediana de $57,8 \mathrm{~kg}$ (IQ 25-75=49,7-61,5), estatura de 1,62 m (IQ 25-75=1,54-1,73) e IMC de $20,4 \mathrm{~kg} / \mathrm{m}^{2}$ (IQ 25-75=18,5-24,2), dos quais dois adolescentes e dois adultos foram classificados como tendo sobrepeso e o restante classificado como eutrófico de acordo com o IMC. Não foi observada correlação entre parâmetros antropométricos e a DMO.

Uma parcela dos pacientes apresentou inadequação do consumo de macronutrientes, sendo $10 \mathrm{com}$ inadequação do consumo de carboidratos e 14 de lipídeos. Em relação à ingestão de micronutrientes, treze pacientes apresentaram baixo consumo de cálcio, onze de magnésio, treze de fósforo e todos os pacientes apresentaram ingestão insuficiente de potássio (Tabelas 2 e 3 ).

Dos indivíduos avaliados, dois não estavam realizando suplementação com FM no momento da inclusão e apresentaram menor ingestão proteica
( 21,7 vs $59,1 \mathrm{~g} / \mathrm{dia})$, de Tyr ( 395,0 vs $3700,0 \mathrm{mg} / \mathrm{dia}$ ), cálcio (154,9 vs $1032,7 \mathrm{mg} / \mathrm{dia})$, fósforo (342,3 vs $848,8 \mathrm{mg} / \mathrm{dia}$ ), magnésio $(142,7$ vs $325,4 \mathrm{mg} / \mathrm{dia})$ e potássio (1874,9 vs 2058,2 mg/dia) e maior ingestão de Phe ( 635,0 vs $400,0 \mathrm{mg} / \mathrm{dia}$ ) quando comparados ao grupo que utilizava FM. A FM foi responsável por suprir a maior parte da ingestão de cálcio, fósforo e magnésio quando comparada a alimentação. Ainda, quando comparada à suplementação isolada de cálcio com mediana de $550 \mathrm{mg} / \mathrm{dia}$ (IQ 25-75=150-1000), a FM permaneceu sendo a maior fonte deste micronutriente para estes pacientes. A origem dos micronutrientes (dieta e FM) está descrita na Tabela 4.

Nenhum dos pacientes apresentou histórico de fraturas. Não foi observada correlação significativa entre a DMO expressa em $\mathrm{g} / \mathrm{cm}^{2}$ e a ingestão de cálcio proveniente da alimentação e FM ( $r=-0,421$; $p=0,118)$. Não foi observada correlação entre a DMO e a ingestão dos demais micronutrientes, fósforo, potássio e magnésio e macronutrientes como carboidratos, proteínas e lipídeos e Phe dietética, bem como valores plasmáticos de Phe, Tyr, cálcio e faixa etária. Não foi encontrada correlação entre a ingestão de cálcio e Phe dietéticos $(r=-0,368$; $p=0,177$ ).

Tabela 1: Densidade mineral óssea e parâmetros bioquímicos $(n=15) .^{a}$

\begin{tabular}{cccc}
\hline & PKU Clássica $(\mathbf{n}=8)$ & PKU Leve $(\mathbf{n}=\mathbf{7})^{\mathrm{a}}$ & $\mathbf{p}$ \\
\hline DMO, $\mathrm{g} / \mathrm{cm}^{2}(\mathrm{n}=15)$ & $0,975(0,906-1,051)$ & $1,040(0,728-1,126)$ & 0,643 \\
Phe sérica, $\mathrm{mg} / \mathrm{dL}(\mathrm{n}=15)$ & $12,9(9,9-14,6)$ & $9,4(5,2-10,9)$ & 0,105 \\
Tyr sérica, $\mathrm{mg} / \mathrm{dL}(\mathrm{n}=15)$ & $0,9(0,5-1,2)$ & $0,8(0,7-0,9)$ & 0,817 \\
Cálcio sérico, $\mathrm{mg} / \mathrm{dL}(\mathrm{n}=13)$ & $9,6(9,1-9,9)$ & $9,70(9,6-10,1)$ & 0,416 \\
\hline
\end{tabular}

DMO: Densidade mineral óssea; Phe: Fenilalanina; Tyr: Tirosina; ${ }^{2} D a d o s$ apresentados em mediana da mediana (Intervalo Interquartil 25-75); PKU: Fenilcetonúria.

Tabela 2: Análise da ingestão de macronutrientes em 15 pacientes com Fenilcetonúria*.

\begin{tabular}{cccccc}
\hline Paciente & Idade (anos) & Calorias (kcal) & Carboidratos $\mathbf{g})$ & Proteínas $\mathbf{( g )}$ & Lipídeos $\mathbf{( g )}$ \\
\hline 1 & 17 & 1321,4 & 237,3 & 61,7 & 14,8 \\
2 & 15 & 850,3 & 160,1 & 39,2 & 8,9 \\
3 & 15 & 1213,5 & 178,1 & 64,6 & 25,1 \\
4 & 26 & 873,9 & 153,4 & 47,6 & 8,7 \\
5 & 15 & 1343,4 & 259,1 & 59,0 & 11,9 \\
6 & 15 & 1141,7 & 178,9 & 64,4 & 17,7 \\
7 & 14 & 1464,8 & 297,6 & 55,3 & 9,1 \\
8 & 23 & 1360,4 & 206,1 & 43,5 & 41,4 \\
9 & 28 & 1099,9 & 229,4 & 25,9 & 11,3 \\
10 & 16 & 1485,3 & 337,2 & 82,8 & 29,2 \\
11 & 20 & 841,4 & 122,5 & 62,4 & 12,9 \\
12 & 18 & 834,4 & 143,5 & 42,3 & 11,0 \\
13 & 8 & 1216,7 & 219,0 & 59,1 & 11,9 \\
14 & 15 & 1151,4 & 224,2 & 17,5 & 20,7 \\
15 & 17 & 1126,7 & 161,6 & 68,8 & 24,5
\end{tabular}

*Dados referentes ao consumo diário de macronutrientes obtidos por recordatório alimentar de 24 horas sem considerar a ingestão de suplementos. 
Tabela 3: Análise da ingestão de micronutrientes e aminoácidos em 15 pacientes com Fenilcetonúria*.

\begin{tabular}{ccccccc}
\hline Paciente & $\begin{array}{c}\text { Cálcio } \\
(\mathbf{m g})\end{array}$ & $\begin{array}{c}\text { Potássio } \\
(\mathbf{m g})\end{array}$ & $\begin{array}{c}\text { Fósforo } \\
(\mathbf{m g})\end{array}$ & $\begin{array}{c}\text { Magnésio } \\
(\mathbf{m g})\end{array}$ & $\begin{array}{c}\text { Fenilalanina } \\
(\mathbf{m g})\end{array}$ & $\begin{array}{c}\text { Tirosina } \\
(\mathbf{m g})\end{array}$ \\
\hline 1 & 1211,2 & 2816,1 & 933,6 & 388,1 & 360 & 3040 \\
2 & 1032,7 & 1763,9 & 735,4 & 250,7 & 310 & 2750 \\
3 & 920,9 & 1274,4 & 776,3 & 277,6 & 520 & 4890 \\
4 & 847,2 & 1308,9 & 682,6 & 256,7 & 370 & 3700 \\
5 & 718,4 & 2673,8 & 1007,3 & 428,9 & 2100 & 2880 \\
6 & 942,4 & 2332,2 & 790,7 & 299,9 & 430 & 4850 \\
7 & 1183,2 & 2055,6 & 862,1 & 325,4 & 220 & 4770 \\
8 & 556,1 & 1953,9 & 528,6 & 168,6 & 400 & 2020 \\
9 & 203,2 & 1244,3 & 282,6 & 115,2 & 610 & 380 \\
10 & 1267,9 & 2105,9 & 982,5 & 342,6 & 500 & 5730 \\
11 & 1151,3 & 2058,2 & 848,8 & 343,4 & 310 & 5390 \\
13 & 796,6 & 1278,1 & 625,4 & 244,3 & 350 & 3680 \\
15 & 1661,2 & 2475,7 & 1536,2 & 371,8 & 450 & 3150 \\
\end{tabular}

*Dados referentes ao consumo diário de micronutrientes e aminoácidos obtidos por recordatório alimentar de 24 horas sem considerar a ingestão de suplementos.

Tabela 4: Micronutrientes provenientes da alimentação e fórmula metabólica ( $n=13)$.

\begin{tabular}{cccc}
\hline & Alimentação & Fórmula Metabólica $^{\text {a }}$ & Total* $^{*}$ \\
\hline Cálcio, mg/dia & $161,9(95,2-213,4)$ & $901,8(342,7-1028,1)$ & $1032,7(803,2-1661,2)$ \\
Fósforo, mg/dia & $255,9(166,4-339,6)$ & $594,0(236,7-708,9)$ & $790,7(625,4-982,5)$ \\
Potássio, mg/dia & $1244,3(1136,9-1976,1)$ & $808,2(307,1-921,3)$ & $2058,2(1308,9-2505,4)$ \\
Magnésio, mg/dia & $92,3(80,1-165,3)$ & $206,5(85,5-228,0)$ & $299,9(244,3-371,8)$ \\
\hline
\end{tabular}

aDados apresentados em mediana (Intervalo Interquartil 25-75); *Dietary Reference Intakes (DRIs) ${ }^{17}$ : Cálcio (RDA): Crianças 4-8 anos: $1000 \mathrm{mg} / \mathrm{dia}$; Masculino: 9-13 anos: $1300 \mathrm{mg} / \mathrm{dia}, 14-18$ anos: $1300 \mathrm{mg} / \mathrm{dia}, 19-30$ anos: $1000 \mathrm{mg} / \mathrm{dia} ;$ Feminino: 9-13 anos: $1300 \mathrm{mg} / \mathrm{dia}$, 14-18 anos: $1300 \mathrm{mg} / \mathrm{dia}, 19-30$ anos: $1000 \mathrm{mg} / \mathrm{dia}$; Fósforo (RDA): Crianças 4-8 anos: $500 \mathrm{mg} / \mathrm{dia}$; Masculino: 9-13 anos: $1250 \mathrm{mg} / \mathrm{dia}$, 14-18 anos: $1250 \mathrm{mg} / \mathrm{dia}, 19-30$ anos: $700 \mathrm{mg} / \mathrm{dia} ;$ Feminino: 9-13 anos: $1250 \mathrm{mg} / \mathrm{dia}, 14-18$ anos: $1250 \mathrm{mg} / \mathrm{dia}, 19-30 \mathrm{anos:} 700 \mathrm{mg} / \mathrm{dia}$; Potássio (Al): Crianças 4-8 anos: 3,8 g/dia; Masculino: 9-13 anos: 4,5 g/dia, 14-18 anos: 4,7 g/dia, 19-30 anos: 4,7 g/dia; Feminino: 9-13 anos: 4,5 g/dia, $14-18$ anos: 4,7 g/dia, 19-30 anos: 4,7 g/dia; Magnésio (RDA): Crianças 4-8 anos: $130 \mathrm{mg} / \mathrm{dia}$; Masculino: 9-13 anos: $240 \mathrm{mg} / \mathrm{dia}$, 14-18 anos: $410 \mathrm{mg} / \mathrm{dia}, 19-30$ anos: $400 \mathrm{mg} / \mathrm{dia}$; Feminino: 9-13 anos: $240 \mathrm{mg} / \mathrm{dia}, 14-18$ anos: $360 \mathrm{mg} / \mathrm{dia}, 19-30$ anos: $310 \mathrm{mg} / \mathrm{dia}$.

\section{DISCUSSÃO}

Este estudo avaliou a DMO e sua correlação com parâmetros nutricionais, antropométricos e bioquímicos em uma amostra de indivíduos jovens com PKU em tratamento. A redução da DMO não foi comum nos pacientes avaliados, o que não está de acordo com revisão sistemática e metanálise a qual sugeriu um aumento do risco de desenvolvimento de osteoporose nessa população².

O presente estudo não observou histórico de fraturas nos indivíduos avaliados. Uma revisão sistemática que incluiu 16 estudos demonstrou uma taxa de ocorrência de fraturas de cerca de $20 \%$ em indivíduos com PKU ${ }^{19}$. Demirdas et al. ${ }^{20}$ avaliaram 60 indivíduos com PKU com mediana de idade de 13 anos, demonstrando uma taxa de fraturas de cerca de $41,7 \%$. Este mesmo estudo, contudo, observou que as taxas de fraturas ao longo da vida em indivíduos com PKU se assemelham às de indivíduos normais. Hansen et al. ${ }^{21}$ sugerem que a análise da ocorrência de fraturas deve ser realizada em indivíduos com e sem PKU pareados por idade e sexo, considerando que a taxa de ocorrência de fraturas e o motivo pelo qual ocorrem difere entre indivíduos jovens e adultos, bem como a taxa de ocorrência de fraturas em homens é maior do que em mulheres.

Estudos sugerem não haver correlação entre níveis séricos de Phe e alteração na DMO em indivíduos com $\mathrm{PKU}^{2,11,19}$, o que está de acordo com nossos achados. No presente estudo não foi encontrada também correlação entre a DMO e a ingestão de cálcio proveniente da alimentação e fórmula metabólica.

Apenas um paciente apresentou níveis de Phe fora do valor alvo em tratamento, sendo a DMO classificada como adequada para a idade. Um estudo 
clínico realizado com 53 indivíduos com PKU em tratamento e com idade média de 16 anos, não mostrou diferença significativa entre a DMO de indivíduos com valores de Phe plasmática acima e abaixo do valor alvo em tratamento ${ }^{14}$. Por outro lado, crianças e adolescentes com PKU considerados não-aderentes ao tratamento, apresentaram maiores valores de Phe plasmática e menor ingestão de cálcio, bem como menor valor para DMO quando comparados a indivíduos considerados aderentes. Este mesmo estudo demonstrou que indivíduos considerados não-aderentes ao tratamento apresentaram idade óssea maior que a idade cronológica, porém este dado não foi avaliado no presente estudo ${ }^{22}$.

Alterações de marcadores de turnover ósseo estão relacionadas com fatores como a maturidade sexual, sendo maiores os níveis de marcadores de formação óssea durante períodos de crescimento e desenvolvimento como infância e adolescência ${ }^{23}$. Em uma revisão sistemática e metanálise foi observado que indivíduos com PKU apresentaram alterações de marcadores de formação e reabsorção óssea, porém não foi observada correlação com níveis séricos de $\mathrm{Phe}^{2}$. Em contrapartida achados de Nagasaka et al..$^{24}$ demonstraram aumento dos níveis de marcadores de reabsorção óssea em 34 indivíduos com PKU quando comparados ao grupo controle. Outro estudo que avaliou 45 indivíduos com PKU com média de idade de 13 anos, demonstrou que indivíduos que não atingiram a maturação sexual apresentaram níveis aumentados de marcadores de formação e reabsorção óssea quando comparados a indivíduos que já atingiram a mesma ${ }^{25}$. O presente estudo foi constituído por uma população jovem e predominantemente de indivíduos puberais ou pós-puberais, no entanto marcadores de turnover ósseo não foram avaliados e não foi encontrada correlação entre a idade e $\mathrm{DMO}$, em consonância com os achados descritos por Modan-Moses et al. ${ }^{11}$.

Parâmetros antropométricos como peso, estatura e IMC não apresentaram correlação com a DMO. Dados semelhantes foram observados em um estudo realizado por Modan-Moses et al. ${ }^{11} \mathrm{com} 31$ indivíduos adultos com PKU, não havendo correlação entre a $\mathrm{DMO}$ e IMC. Por outro lado um estudo demonstrou correlação positiva entre o valor do escore Z da DMO e valores de escore $Z$ para peso, estatura e IMC em indivíduos com PKU $<18$ anos ${ }^{14}$. No presente estudo, valores plasmáticos de Phe, Tyr e cálcio não apresentaram correlação com a DMO, estando de acordo com dados descritos na literatura que demonstram não haver correlação entre valores séricos de Phe e achados de DMO $11,14,24$.
O tratamento da PKU consiste em dieta restrita em Phe e utilização de FM, sendo observado no presente estudo que pacientes que não utilizam FM apresentaram redução da ingestão calórica, proteica e lipídica, bem como de nutrientes como cálcio, fósforo, magnésio e Tyr. Tais dados confirmam os achados de Oliveira et al. $(2016)^{8}$ de que a FM é responsável por suprir a maior parte das necessidades proteicas diárias, fornecendo também quantidade adequadas de micronutrientes. Estudos demonstram que indivíduos com PKU crianças, adolescentes e adultos considerados não aderentes ao tratamento apresentam redução do consumo de nutrientes como cálcio e proteínas ${ }^{11,22}$.

A ingestão de Phe e cálcio, bem como dos demais macronutrientes e micronutrientes não apresentou correlação com a DMO. Em seu estudo Modan-Moses et al. ${ }^{11}$ avaliaram a ingestão alimentar de pacientes com PKU por meio de 3 diários alimentares, não sendo observada correlação entre a $\mathrm{DMO}$ e a ingestão dietética de cálcio e proteína. Em seu estudo Evans et al. ${ }^{9}$ compararam a ingestão alimentar e parâmetros bioquímicos, sendo observado que a ingestão de micronutrientes acima dos valores recomendados não foi capaz de influenciar marcadores nutricionais bioquímicos, não havendo correlação entre estes, exceto para selênio. Apesar da adequada ingestão dietética de zinco, crianças com PKU menores de 11 anos apresentaram concentrações plasmáticas deste micronutriente abaixo dos valores recomendados ${ }^{9}$. Baixas concentrações de cálcio e magnésio foram observadas em crianças com PKU, entretanto foram observados valores normais para fósforo, zinco e cobre quando comparadas ao grupo controle ${ }^{9,13}$. Estudos demonstram que a ingestão de nutrientes pode não refletir o status biológico de micronutrientes em pacientes com $\mathrm{PKU}^{5,9}$.

\section{CONCLUSÃO}

A redução da DMO bem como a ocorrência de fraturas não foi comum entre os indivíduos avaliados. Dados detalhados sobre o tempo de suplementação e análise da DMO previamente alterada poderiam contribuir para avaliar o efeito da ingestão dietética sobre o metabolismo ósseo. Não foi observada correlação entre valores séricos de Phe e as variáveis avaliadas, no entanto a maior parte dos pacientes apresentou bom controle dos níveis deste marcador.

Dessa forma, são necessários estudos com maior tamanho amostral e um estudo mais aprofundado do consumo alimentar, por meio da realização de 
recordatórios alimentares seriados ao longo do ano que antecede a DEXA. Dosagens séricas de cálcio, fósforo, potássio, vitamina $\mathrm{D}, \mathrm{Phe}, \mathrm{PTH}$ e marcadores de turnover ósseo constituem marcadores importantes para avaliação do metabolismo ósseo e devem ser realizados preferencialmente na mesma data da realização da DEXA. A análise de valores de Phe plasmática nos períodos da infância e adolescência, nos quais ocorre maior crescimento e desenvolvimento ósseo, poderiam contribuir para compreender o efeito da ingestão dietética e níveis séricos de Phe sobre o metabolismo ósseo, a fim de prevenir o desenvolvimento de alterações na DMO em pacientes com $\mathrm{PKU}$.

\section{Agradecimentos}

Agradecimentos ao Hospital de Clínicas de Porto Alegre, Serviço de Genética Médica-HCPA, Equipe de Estatística-HCPA e a Soraia Poloni pelo espaço e conhecimento proporcionados. Este estudo recebeu apoio financeiro FIPE-HCPA. O conteúdo científico declarado no artigo é de responsabilidade dos autores.

\section{Fonte de Financiamento}

Fundo de Incentivo a Pesquisa e Eventos (FIPE)Hospital de Clínicas de Porto Alegre (HCPA).

\section{Conflito de Interesse}

Os autores declaram não ter conflito de interesse.

\section{REFERÊNCIAS}

1. Blau N, Van Spronsen FJ, Levy $\mathrm{HL}$. Phenylketonuria. Lancet. 2010;376(9750):1417-27. http://dx.doi. org/10.1016/S0140-6736(10)60961-0. PMid:20971365.

2. Demirdas S, Coakley KE, Bisschop $\mathrm{PH}$, Hollak CEM, Bosch AM, Singh $\mathrm{RH}$. Bone health in phenylketonuria: a systematic review and meta-analysis. Orphanet J Rare Dis. 2015;10(1):17. http://dx.doi.org/10.1186/s13023-0150232-y. PMid:25758373.

3. Al Hafid N, Christodoulou J. Phenylketonuria: a review of current and future treatments. Transl Pediatr. 2015;4(4):304-17. PMid:26835392.

4. Monteiro LTB, Cândido LMB. Fenilcetonúria no Brasil: evolução e casos. Rev Nutr. 2006;19(3):381-7. http://dx.doi.org/10.1590/S141552732006000300009 .

5. Robert M, Rocha J, van Rijn M, Ahring K, Bélanger-Quintana A, MacDonald $A$, et al. Micronutrient status in phenylketonuria. Mol Genet Metab. 2013;110(Suppl):S6-17. http://dx.doi. org/10.1016/j.ymgme.2013.09.009. PMid:24113686.

6. Singh RH, Rohr F, Frazier D, Cunningham A, Mofidi S, Ogata $B$, et al. Open Recommendations for the nutrition management of phenylalanine hydroxylase deficiency. Genet Med. 2014;16(2):121-31. http:// dx.doi.org/10.1038/gim.2013.179. PMid:24385075.

7. Macleod EL, Ney DM. Nutritional management of phenylketonuria. Annales Nestlé. 2010;68(2):58-69. PMid:22475869.
8. Oliveira FP, Mendes RH, Dobbler PT, Mai V, Pylro VS, Waugh SG, et al. Phenylketonuria and gut microbiota: a controlled study based on nextgeneration sequencing. PLoS One. 2016;11(6):e0157513. http://dx.doi. org/10.1371/journal.pone.0157513. PMid:27336782.

9. Evans S, Daly A, MacDonald J, Preece MA, Santra S, Vijay S, et al. The micronutrient status of patients with phenylketonuria on dietary treatment: an ongoing challenge. Ann Nutr Metab. 2014;65(1):42-8. http://dx.doi.org/10.1159/000363391. PMid:25196394.

10. McMurry MP, Chan GM, Leonard $\mathrm{CO}$, Ernst SL. Bone mineral status in children with phenylketonuria-relationship to nutritional intake and phenylalanine control. Am J Clin Nutr. 1992;55(5):997-1004. http:// dx.doi.org/10.1093/ajcn/55.5.997. PMid:1570811.

11. Modan-Moses D, Vered I, Schwartz G, Anikster Y, Abraham Y, Segev $\mathrm{R}$, et al. Peak bone mass in patients with phenylketonuria. J Inherit Metab Dis. 2007;30(2):202-8. http://dx.doi. org/10.1007/s10545-007-0462-9. PMid:17347917.

12. Crujeiras V, Aldámiz-Echevarría L, Dalmau J, Vitoria I, Andrade F, Roca I, et al. Vitamin and mineral status in patients with hyperphenylalaninemia. Mol Genet Metab. 2015;115(4):14550. http://dx.doi.org/10.1016/j. ymgme.2015.06.010. PMid:26123187.

13. Hillman L, Schlotzhauer C, Lee D, Grasela J, Witter S, Allen S, et al. Decreased bone mineralization in children with phenylketonuria under treatment. Eur J Pediatr.
1996;155(S1 Suppl 1):S148-52. http:// dx.doi.org/10.1007/PL00014234. PMid:8828633.

14. De Groot MJ, Hoeksma M, Van Rijn M, Slart RHJA, Van Spronsen FJ. Relationships between lumbar bone mineral density and biochemical parameters in phenylketonuria patients. Mol Genet Metab. 2012;105(4):566-70. http://dx.doi. org/10.1016/j.ymgme.2012.01.006. PMid:22300845.

15. Brasil. Ministério da Saúde. Portaria n 1307 , de 22 de novembro de 2013. Aprova o Protocolo Clínico e Diretrizes Terapêuticas da Fenilcetonúria. Diário Oficial da União. 25 Nov 2013.

16. Nalin T, Perry IDS, Refosco LF, Netto CBO, Souza CFM, Vieira TA, et al. Fenilcetonúria no Sistema Único de Saúde: Avaliação de Adesão ao Tratamento em um Centro de Atendimento do Rio Grande do Sul. Revista HCPA. 2010;30:225-32.

17. Institute of Medicine. Dietary Reference Intakes for Calcium and Vitamin D. Washington: The National Academies Press; 2011.

18. Brandão CMA, Camargos BM, Zerbini CA, Plapler PG, Mendonça LMC, Albergaria B, et al. 2008 Official Positions of the Brazilian Society for Clinical Densitometry--SBDens. Arq Bras Endocrinol Metabol. 2009;53(1):107-12. PMid:19347193.

19. Hansen KE, Ney D. A systematic review of bone mineral density and fractures in phenylketonuria. J Inherit Metab Dis. 2014;37(6):875-80. http:// dx.doi.org/10.1007/s10545-014-97352. PMid:25005329. 
20. Demirdas S, Van Spronsen FJ, Hollak CEM, Van Der Lee JH, Bisschop $\mathrm{PH}$, Vaz FM, et al. Micronutrients, essential fatty acids and bone health in phenylketonuria. Ann Nutr Metab. 2017;70(2):111-21. http:// dx.doi.org/10.1159/000465529. PMid:28334709.

21. Hansen KE, Binkley N, Ney DM. High fracture rates in young patients with phenylketonuria. Ann Nutr Metab. 2018;72(1):1-2. http:// dx.doi.org/10.1159/000484617. PMid:29145199.

22. Mendes $A B$, Martins FF, Cruz WMS, Silva LE, Abadesso CBM, Boaventura GT. Bone development in children and adolescents with PKU. J Inherit Metab Dis. 2012;35(3):425-30. http://dx.doi. org/10.1007/s10545-011-9412-7. PMid:22076426.

23. Rauchenzauner $M$, Schmid A, Heinz-Erian P, Kapelari K, Falkensammer G, Griesmacher A, et al. Sex- and Age-Specific Reference Curves for Serum Markers of Bone Turnover in Healthy Children from 2 Months to 18 Years. J Clin Endocrinol Metab. 2007;92(2):443-9. http:// dx.doi.org/10.1210/jc.2006-1706. PMid:17105843.
24. Nagasaka $H$, Tsukahara $H$, Takatani T, Sanayama Y, Takayanagi M, Ohura $\mathrm{T}$, et al. Cross-sectional study of bone metabolism with nutrition in adult classical phenylketonuric patients diagnosed by neonatal screening. $J$ Bone Miner Metab. 2011;29(6):73743. http://dx.doi.org/10.1007/s00774011-0276-6. PMid:21594581.

25. Adamczyk P, Morawiec-Knysak A, Płudowski P, Banaszak B, Karpe J, Pluskiewicz W. Bone metabolism and the muscle-bone relationship in children, adolescents and young adults with phenylketonuria. J Bone Miner Metab. 2011;29(2):236-44. http://dx.doi.org/10.1007/s00774010-0216-x. PMid:20706750.

Recebido: 2 dez, 2018 Aceito: 26 abr, 2019 